\title{
Industria de la información y piratería digital en México: análisis económico de la protección de los derechos de autor
}

Manuel Gerardo Chávez Ángeles*

Patricia S. Sánchez Medina**

Artículo recibido:

28 de julio de 2014.

Artículo aceptado:

17 de abril de 2015.

\section{Resumen}

El artículo hace un análisis económico de la protección de la propiedad intelectual en México. A través del desarrollo de un modelo de teoría de juegos de "guerra de desgaste", construye distintos escenarios de organización industrial para el negocio de la información. Se evalúan los efectos en la dinámica industrial derivados de la existencia de un régimen de protección de propiedad intelectual, en particular los derechos de autor.

* Doctor en Ciencias en Conservación y Aprovechamiento de Recursos Naturales por el Instituto Politécnico Nacional (IPN), maestro de Administración Pública y Desarrollo Internacional por la Universidad de Harvard, candidato al Sistema Nacional de Investigadores (SNI) y profesor investigador de la Universidad de la Sierra Sur. mchavez@unsis.edu.mx; manuelchavezangeles@hotmail.com

** Doctora en Ciencias en Conservación y Aprovechamiento de Recursos Naturales por el Instituto Politécnico Nacional (IPN), miembro del Sistema Nacional de Investigadores (SNI) nivel I, profesora investigadora del IPN, CIIDIR, Unidad Oaxaca. psanchez@ipn.mx; ms_287506@yahoo.com.mx

INVESTIGACIÓN BIBLIOTECOLÓGICA, vol. 31, núm. 71, enero/abril, 2017, México, ISSN: 0187-358X, pp. 53-72 
Se encuentra que con éstos la estrategia dominante de la industria establecida es combatir la piratería esperando obtener beneficios monopólicos. Utilizando datos de la Encuesta de Uso de Tiempo (ENUT) del INEGI, el artículo desarrolla un perfil del consumo de medios y bienes culturales de la población mexicana y del acceso a Internet. Se encuentra que el $21.1 \%$ de la población mexicana está en situación de pobreza digital extrema, sin acceso a conectividad digital alguna.

Palabras clave: Análisis económico del derecho; Comunes de información; Propiedad intelectual; Teoría de juegos.

\section{Abstract}

Information Industries and Digital Piracy: An Economic Analysis of Intellectual Property Law Manuel Gerardo Chávez-Ángeles and Patricia S. Sánchez-Medina

The article presents an economic analysis of the protection of intellectual property in Mexico. The article develops a war of attrition game theory model, presenting different scenarios of industrial organization for information industries. The article shows that with the existence of copyright, the dominant strategy of industry is to combat piracy for the purpose of securing monopoly power. Using data from the National Survey on the Use of Time (INEGI, by its Spanish-language acronym), of the article develops a profile of media and cultural products consumption and access to digital technologies. In Mexico, $21.1 \%$ of the population is in extreme digital poverty.

Keywords: Economic Analysis of Law; Common Information; Copyright; Game Theory.

\section{Introducción}

La tecnología digital ha ocasionado importantes alteraciones en las industrias de la información, en particular en la producción y distribución musical. El desarrollo del formato mp3 cambió la forma de almacenar música para siempre. El mp3, creado por ingenieros de la compañía alemana Fraunhofer 
Gesellshaft, es una abreviación del Motion Picture Experts Group-Layer 3. Un formato compactador de audio que genera una calidad de sonido cercana a la de los discos compactos en un tamaño 10 o 20 veces menor. Por ejemplo: el álbum sencillo Hound Dog, de Elvis Presley, en disco compacto requiere 24 megabytes de espacio en un disco duro, pero cuando es convertido a mp3 el espacio requerido es de tan sólo 2 megabytes. Además, en una conexión de Internet de 28.8 kilobits por segundo, el disco compacto de Hound Dog tomaría al menos una hora y media para ser descargado en otra computadora, mientras que si el archivo fuera previamente convertido a mp3 tomaría aproximadamente ocho minutos y medio. ${ }^{1}$

En términos de protección de los derechos de autor, la industria formal tomó la ofensiva a partir del año 2000 cuando Metallica demandó legalmente a Napster. Desde entonces, las compañías disqueras han buscado preservar su modelo de negocios tomando acciones legales contra los mayores sitios de distribución de archivos digitales de Internet. El sitio web mp3.com fue encontrado culpable de violar los derechos de autor de la asociación de la Industria de la Grabación de Estados Unidos (RIAA, por sus siglas en inglés) por una corte de distrito de Estados Unidos. Asimismo, un juez federal, también en Estados Unidos, rechazó la apelación de Napster.com y ordenó que dejara de distribuir material protegido bajo la ley de derechos de autor. ${ }^{2}$

La prensa escrita de los países desarrollados también ha experimentado importantes cambios derivados de la migración de sus lectores, del papel a los soportes digitales. En el ámbito académico se han realizado distintas tipologías del acceso a la prensa digital. ${ }^{3}$ En mayo de 2009, el Senado de Estados Unidos realizó una audiencia sobre el futuro de los periódicos. La Declaración de la Mayoría, firmada por John D. Rockefeller IV, decía:

Durante aproximadamente los últimos seis meses, los periódicos de circulación diaria han declinado en 7 por ciento. Durante aproximadamente el año pasado las compañías de medios han tenido que hacer recortes de 41000 empleados. El resultado inevitable es menos reportajes, menos noticias y menos cobertura de nuestras comunidades e intereses en casa y en el extranjero.

A partir de estos hechos podemos inferir que el modelo periodístico que sirvió tan bien en el pasado está ahora en problemas. El futuro del periodismo es digital. Estamos rápidamente migrando de un mundo donde las noticias se imprimen a uno donde las noticias se distribuyen de forma digital a través de una infini-

1 P. Alexander, "Entry Barriers, Release Behavior, and Multi-Product Firms in the Music Recording Industry”, Review of Industrial Organization 9 (1994): 85-98.

2 Alexander, "Entry Barriers, Release Behavior...", 85-98.

3 J. Guallar, E. Abadal y L. Codina, "Sistemas de acceso a la información de prensa digital: tipología y evolución”, Investigación Bibliotecológica 27, no. 61 (2013): 29-52. 
dad de redes en Internet. Hay mucho que celebrar y explorar sobre este cambio: acceso a una vasta gama de ideas y opiniones y actualizaciones minuto a minuto; pero también es causa de preocupación.

$[\ldots]$

Falta de equidad en el acceso a Internet en algunas comunidades es un problema que necesita ser atendido. También hay pérdidas incuantificables. La experiencia cotidiana de abrir un periódico, sentir al tacto sus páginas, y dejar que la mirada dance sobre el papel es un placer que me temo la siguiente generación no podrá conocer. ${ }^{4}$

En mayo de 2012, el Times-Picayune, el diario de mayor tradición en Nueva Orleans, anunció que reduciría la producción de su edición impresa a sólo tres veces a la semana. El mismo día fue anunciada la creación de NOLA Media Group, consorcio que fusiona la edición impresa del Times-Picayune y su sitio asociado NOLA.com. El cambio dejó a Nueva Orleans como la ciudad más grande de Estados Unidos sin un periódico de circulación diaria. Una semana después, sus lectores hicieron manifestaciones para "salvar el periódico".

En un sistema de comunicación existen tres distintas coberturas que en conjunto hacen posible la transmisión de mensajes: 1) los artefactos físicos y canales de redes necesarios para la comunicación; 2) los recursos de información y cultura a partir de los cuales se pueden hacer nuevas aseveraciones; y 3) los recursos lógicos del software y los estándares necesarios para traducir el lenguaje natural en señales que una máquina puede procesar y transmitir. ${ }^{5}$ Los efectos de las tecnologías digitales en las industrias culturales se han dado tanto en el intercambio entre individuos y empresas como al interior de las empresas. En especial en la industria televisiva los soportes digitales han ocasionado importantes cambios en la selección y preservación documental. ${ }^{6}$

Este artículo trata acerca del comercio e intercambio de bienes de la segunda capa y sobre cómo las condiciones en la primera y tercera capa afectan el comercio e intercambio de información y recursos culturales. La hipótesis del artículo es que las tecnología digitales han desatado una "guerra de desgaste" dentro de la industria formalmente establecida, conformada principalmente por grandes empresas transnacionales y múltiples pequeños microempresarios que ven en la piratería digital una oportunidad de negocios. El artículo concluye que la presencia de la piratería digital dependerá del costo

4 U. S. Senate Committee on Commerce, Science and Transportation, The Future of Journalism, Hearing May 6, 2009 (SR-253), Washington D.C. http://commerce.senate.gov.

5 Y. Benkler, The Wealth of Networks, How Social Production Transforms Markets and Freedom (Yale University Press, 2006).

6 J. Caldera y P. Arranz, "Cambios en los métodos de selección documental en los sistemas de información digital en las televisiones”, Investigación Bibliotecológica 27, no. 60 (2013): 15-26. 
de permanecer en la industria. Se encuentra que, mientras exista la promesa de ganancias monopólicas otorgadas por los derechos de autor, la estrategia dominante de la industria será combatir la piratería o exigir al gobierno que lo haga. Asimismo, se argumenta que en las comunidades en situación de pobreza digital las microempresas de piratería son uno de los canales mediante los cuales aquéllas tienen acceso a las industrias culturales.

\section{Modelo de guerra de desgaste en industrias de la información}

A continuación se desarrolla un análisis económico de la protección de la propiedad intelectual, en particular de los derechos de autor. Esto se ubica dentro de la tradición del análisis económico del derecho o law and economics (en su traducción al inglés). En dicha tradición, la racionalidad de los agentes es un supuesto fundamental para el estudio de las leyes; considera agentes racionales que buscan maximizar sus beneficios dadas ciertas restricciones, las cuales pueden ser económicas o legales. Por tanto, se observan las estrategias derivadas de la interacción entre dichos agentes. En este sentido no es un análisis de tipo normativo, sino uno de la conducta de los agentes económicos que evalúan los costos y beneficios acerca de respetar o no respetar la ley. ${ }^{7}$

Uno de los métodos útiles para el estudio del derecho y la economía es la teoría de juegos. La teoría de juegos explica el comportamiento de los actores en diversas situaciones en términos de la maximización de beneficios. Cada actor tiene una estrategia que matemáticamente se expresa como una función de las estrategias de los demás. Los actores tomarán decisiones considerando las decisiones de los demás hasta alcanzar un equilibrio desde el cual no podrán mejorar unilateralmente, a menos de que se muevan simultáneamente con otros mediante una acción concertada. Este equilibrio es llamado equilibrio de Nash, el cual incluye el principio de las expectativas racionales. Si cada jugador espera un determinado equilibrio, entonces la maximización de los beneficios conducirá a alcanzarlo a cumplir con dichas expectativas. Asimismo, la teoría de juegos nos permite encontrar los resultados socialmente óptimos y observar por qué estos equilibrios no están siendo jugados, y también construir esquemas de incentivos para salir de los resultados subóptimos y llegar los mejores. 
La "guerra de desgaste" es un juego estático simple, popular en el estudio de los monopolios. La información puede considerarse como una industria. ${ }^{8}$ De esta forma, en la organización industrial, la guerra de desgaste es llevada a cabo tanto por medios legales como por guerras de precios. En este ámbito, es posible distinguir tres tipos de comportamiento por parte de los propietarios ante la amenaza de la entrada de un rival: ${ }^{?}$

a) Bloquear la entrada: el propietario compite como si no hubiera barreras en la entrada, de tal manera que el mercado deja de ser atractivo para los rivales.

b) Disuadir la entrada: la entrada no puede ser bloqueada indefinidamente, pero los propietarios modifican su comportamiento ocasionalmente para evitar la entrada exitosamente.

c) Acomodar la entrada: el propietario encuentra más rentable dejar que los rivales entren que erigir costosas barreras a la entrada.

Los oligopolistas son afectados por diversas variables que no pueden observar o estimar con precisión: su propia función de costos, la función de costo de sus rivales, el estado de la demanda o el potencial de mercado, además de las decisiones estratégicas de sus rivales. En la medida en que algunas piezas de la información son privadas, deberemos concebir la interacción de mercado como un juego con información asimétrica. ${ }^{10}$

La Figura 1 muestra este comportamiento de forma sencilla, donde D1 y D2 son el costo de bloquear o disuadir la entrada para los piratas y la industria, respectivamente. Las estrategias dominantes dependerán del valor de D1 y D2. Bajo la información asimétrica, D1(D2) y D2(D1) son las estrategias de los piratas y la industria para bloquear o disuadir la entrada, respectivamente.

Supongamos la existencia de una población de individuos y empresas de tamaño $n$ realizando actividades de producción y copia digital de bienes culturales. Estos individuos y empresas pueden ser piratas o miembros de la industria establecida. Sea ๗ el beneficio derivado de la producción digital:

$$
\text { (1) } \varpi_{\mathrm{i}}=p[\mathrm{Q} / n]-c-\mathrm{D}_{\mathrm{i}} ; \varpi_{\mathrm{m}}=p \mathrm{Q}-c
$$

8 S. Armendáriz Sánchez, "La información como industria", Investigación Bibliotecológica 12 (1992): 11-24.

9 J. Tirole, The Theory of Industrial Organization (Cambridge, Massachusetts: The MIT Press, 1995).

10 J. Riley, "Strong Evolutionary Equilibrium and the War of Attrition", Journal of Theoretical Biology 82 (1980): 383-400; D. Kreps y R. Wilson, "Reputation and Imperfect Information", Journal of Economic Theory 27 (1982): 253-279. 
Figura 1. Guerra de desgaste en industrias culturales

\begin{tabular}{|l|l|l|l|}
\hline \multicolumn{2}{|c|}{} & \multicolumn{2}{|l|}{ Industria } \\
\cline { 3 - 4 } \multicolumn{2}{|c|}{} & Bloquear/disuadir & Acomodarse \\
\hline \multirow{2}{*}{ Pirata } & Bloquear/disuadir & Q/2-D1, Q/2- D2 & Q, 0 \\
\cline { 2 - 4 } & Acomodarse & $0, Q$ & 0,0 \\
\hline \multicolumn{2}{|l|}{ La estrategia dominante depende de D1 y D2. } \\
\hline
\end{tabular}

Donde $\varpi_{i}$ son los beneficios para cada firma en equilibrios competitivos y $\varpi_{\mathrm{m}}$ es el beneficio del monopolio; $p$ es el precio de los productos pirata y $\mathrm{Q}$ la demanda total del producto; $c$ es el costo de producción y $n$ es el número total de productores en la industria. La Figura 2 muestra la forma normal para dos firmas.

El número total de productores $n$ (piratas y no-piratas) se alcanzará donde $\varpi=0$ :

(2) $n=p \mathrm{Q} / \mathrm{D}_{i}+c$

Figura 2. Guerra de desgaste en las industrias culturales.

La piratería como actividad empresarial

\begin{tabular}{|l|l|l|l|}
\hline \multicolumn{2}{|c|}{} & \multicolumn{2}{|l|}{ Industria } \\
\cline { 3 - 4 } \multicolumn{2}{|c|}{} & Bloquear/disuadir & Acomodarse \\
\hline \multirow{3}{*}{ Pirata } & Bloquear/disuadir & $\pi_{1,} \pi_{2}$ & $\pi_{m, 0}$ \\
\cline { 2 - 4 } & Acomodarse & $0, \pi_{m}$ & 0,0 \\
\hline \multicolumn{2}{|l}{ La estrategia dominante depende de D1 y D2. }
\end{tabular}

Mientras el costo del bloqueo/disuasión sea mayor, menor será el número de productores. El costo de producción no se considera una variable relevante, dado que se encuentra en descenso desde la invención de la cinta magnética en 1950. De hecho, no hay duda de que el cambio actual en las industrias culturales y el acelerado crecimiento de la piratería se debe al desplome de los costos de producción después de la invención del formato mp3. Los artistas y las compañías disqueras independientes se benefician del cambio en la estructura de costos, principalmente a través del menor costo de producción y distribución. 
Es posible mencionar cuatro elementos de la estructura de mercado que afectan la habilidad de establecer firmas que prevengan que las rentas monopólicas sean erosionadas por la entrada de rivales. Tomando el caso de la música digital, la Tabla 1 muestra los cambios en la estructura de mercado premp3 y postmp3. ${ }^{11}$

Ahora supongamos la existencia de derechos de autor en un juego dinámico con infinitos periodos donde el beneficio para los piratas en el mercado negro está dado por:

$$
\text { (3) } \varpi_{p}=\sum^{0 \mathrm{~T}} p[\mathrm{Q} / n]
$$

Tabla 1. Elementos de la estructura de mercado

\begin{tabular}{|l|c|c|}
\hline & Pre-mp3 & Post-mp3 \\
\hline $\begin{array}{l}\text { Economías de escala: si la escala mínima eficiente (EME) }{ }^{\star} \text { es una porción } \\
\text { significativa de la demanda de la industria, el mercado puede sostener únicamente } \\
\text { un pequeño número de empresas que tengan beneficios súper normales sin } \\
\text { propiciar la entrada de competidores. }\end{array}$ & Sí & N0 \\
\hline $\begin{array}{l}\text { Ventaja absoluta en costos: las empresas establecidas pueden estar en posesión } \\
\text { de técnicas superiores de producción aprendidas a lo largo de la experiencia } \\
\text { (aprender haciendo) o mediante la investigación y el desarrollo (R\&D). También } \\
\text { pueden haber acumulado un capital que reduce sus costos de producción o haber } \\
\text { captado a importantes proveedores y de esta manera bloquear la entrada a los } \\
\text { competidores. }\end{array}$ & Sí & N0 \\
\hline $\begin{array}{l}\text { Ventajas en la diferenciación del producto: los incumbentes pueden haber } \\
\text { patentado innovaciones, captado los nichos correctos en el espacio de productos o } \\
\text { gozar de la lealtad de los consumidores. }\end{array}$ & SÍ & N0 \\
\hline $\begin{array}{l}\text { Requerimientos de capital: los competidores entrantes pueden tener dificultades de } \\
\text { encontrar financiamiento para sus inversiones debido al riesgo de los acreedores. }\end{array}$ & SÍ & N0 \\
\hline *EME: volumen de producción que minimiza el coste medio en relación con el nivel de la demanda. \\
\hline
\end{tabular}

Y el beneficio para la industria está dado por:

$$
\text { (4) } \varpi_{\mathrm{I}}=\varpi_{c}+\varpi_{m}=\sum^{0 \mathrm{~T}} p[\mathrm{Q} / n]+\sum^{\mathrm{T}}{ }^{\infty} p \mathrm{Q}
$$

Donde $\varpi_{c} y \varpi_{m}$ son los ingresos en un mercado competitivo y en los ingresos monopólicos, respectivamente; $\mathrm{T}$ es el periodo en el cual el competidor abandona el mercado. 
Figura 3. Guerra de desgaste en las industrias culturales. La piratería como actividad emprendedora

\begin{tabular}{|c|c|c|c|}
\hline & \multicolumn{2}{|l|}{ Industria } \\
\hline & & $\begin{array}{c}\text { Bloquear/disuadir } \\
D p,-D i\end{array}$ & Acomodarse \\
\hline \multirow{2}{*}{ Pirata } & Bloquear/disuadir & $\pi_{P-\mathrm{Dp},} \pi_{I}-\mathrm{Di}$ & $\pi_{P, 0}$ \\
\hline & Acomodarse & $0, \pi_{I}$ & 0,0 \\
\hline
\end{tabular}

Claramente ${ }_{\mathrm{I}} \geq{ }_{\text {p. }}$ En este sentido no importa qué tan alejado se encuentre T siempre y cuando haya un potencial infinito de ganancias monopólicas después de T. El potencial de ganancias monopólicas infinitas está dado por la ley de protección de derechos de autor. Una vez más, son los costos $\mathrm{D}_{p}$ y $\mathrm{D}_{\mathrm{I}}$ los que pueden cambiar las estrategias dominantes de ambos (piratas y no piratas) para entrar al mercado.

Utilizando la ecuación 4 se realizó una simulación de programación lineal, suponiendo que $n$ decrece de manera constante y que $\mathrm{D}_{\mathrm{I}}$ se incrementa constantemente. Los resultados (Figura 4) sugieren que (al menos bajo estos supuestos) la industria tendrá que asumir algunas pérdidas económicas antes de obtener ganancias monopólicas. Si estas pérdidas son suficientemente profundas, la empresa podrá preferir acomodarse antes de obtener dichas ganancias.

Figura 4. A. Beneficios de la industria sin costos de bloqueo/disuasión

$(\mathrm{DI}=0)$ y decremento constante de la población pirata (N)

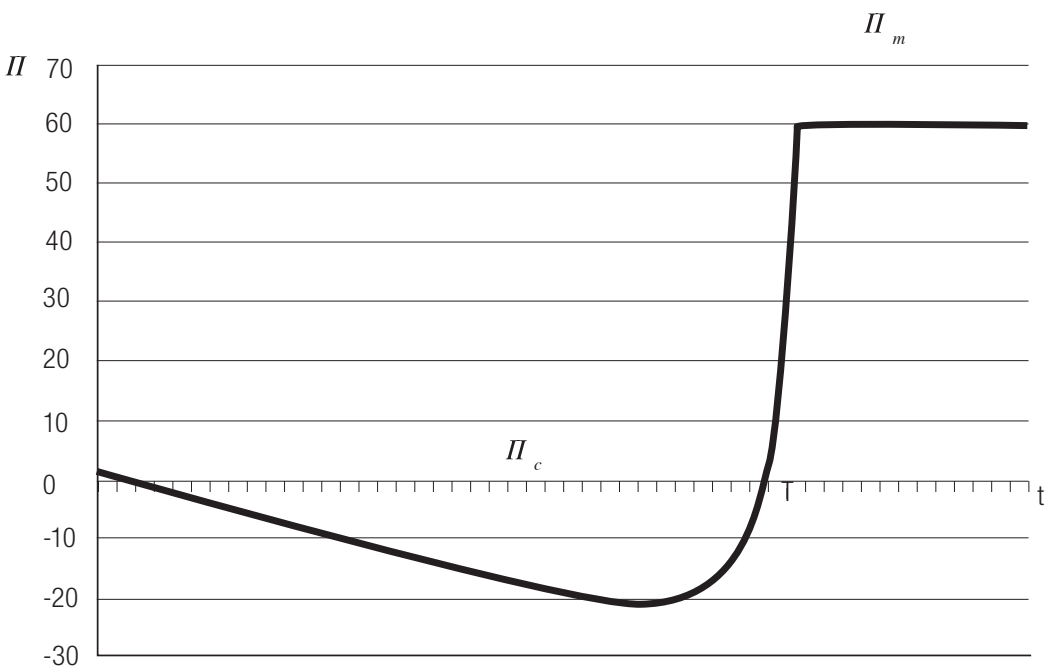


Figura 4. B. Beneficios de la industria con un decremento constante de la población pirata y un incremento constante en el costo de bloqueo/disuasión

(Dl>0; Dl'>0)

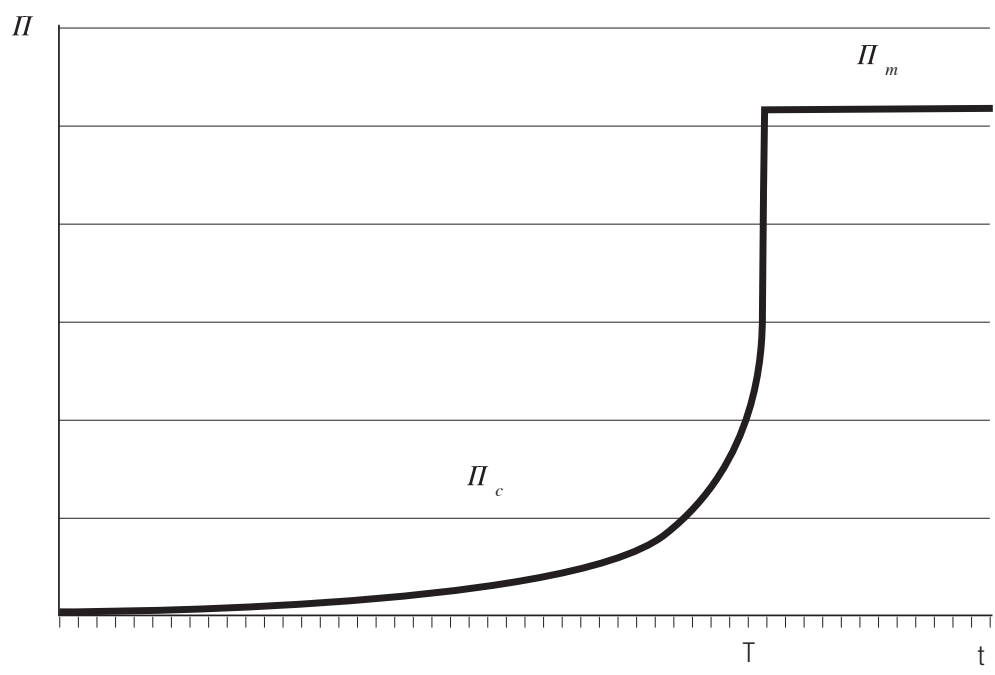

Discusión: propiedad intelectual y consumo de medios en México

A pesar de que las tecnologías digitales tienen efectos globales, su impacto no ha estado distribuido de forma equitativa en distintas partes del mundo. El grado de conectividad es un elemento de gran importancia para definir las estrategias corporativas y ciudadanas de comercialización y de acceso a la información. México es un caso particular donde aún encontramos hogares en situación de pobreza digital. Utilizando datos de la Encuesta Nacional de Uso del Tiempo (ENUT) del Instituto de Estadística, Geografía e Informática (INEGI), se estima que el $60.7 \%$ de los hogares son propietarios de al menos un teléfono celular, siendo la tecnología móvil el principal determinante de la inclusión digital; sin embargo, el 21.1\% de los hogares se encuentran todavía en un completo apagón mediático o pobreza digital extrema, sin acceso a teléfonos fijos o celulares, Internet o TV por cable.

Tabla 2. Difusión de la conectividad por hogar (\%)

\begin{tabular}{|l|l|l|l|l|}
\hline Teléfono & Móvil & TV p/cable & Internet & Pobreza digital extrema* $^{*}$ \\
\hline 40.3 & 60.7 & 27.7 & 18.1 & 21.1 \\
\hline
\end{tabular}


No obstante la pobreza digital, la población mexicana gasta un 58.9\% de las horas dedicadas al ocio en actividades relacionadas con los medios masivos de información, tales como ver TV, navegar en Internet, leer revistas y libros; el 28.1\% de las horas disponibles son asignadas a la convivencia social; el 6.7\% es ocupado en practicar algún deporte; el 4.2\% se destina a juegos y aficiones; y tan sólo el $2.1 \%$ es dedicado a la asistencia a eventos culturales, deportivos y de entretenimiento.

Tabla 3. Demanda de ocio

\begin{tabular}{|l|l|l|l|}
\hline Tiempo dedicado a diferentes actividades (\%) & Horas totales & Mujeres & Hombres \\
\hline $\begin{array}{l}\text { Convivencia social } \\
\text { (ej.: fiestas, misas, desfiles, mítines, hablar por teléfono, chatear, } \\
\text { etcétera). }\end{array}$ & 28.1 & 30.4 & 25.8 \\
\hline $\begin{array}{l}\text { Eventos culturales, deportivos y de entretenimiento } \\
\text { (ej.: museos, parques, ferias, estadios, exposiciones, cine, teatro, } \\
\text { conciertos, etcétera). }\end{array}$ & 2.1 & 2 & 2.2 \\
\hline $\begin{array}{l}\text { Participación en juegos y aficiones } \\
\text { (ej.: tocar un instrumento musical, bailar, pintar o realizar artes } \\
\text { plásticas, gráficas, literarias o escénicas, videojuegos, juegos } \\
\text { de mesa o azar, juegos con mascotas o juguetes). }\end{array}$ & 4.2 & 3.2 & 5.1 \\
\hline $\begin{array}{l}\text { Deportes y ejercicio físico } \\
\text { (ej.: fútbol, basquetbol, natación, box, karate, correr, caminar, patinar, } \\
\text { andar en bicicleta, etcétera). }\end{array}$ & 6.7 & 4.9 & 8.4 \\
\hline $\begin{array}{l}\text { Utilización de medios masivos de comunicación } \\
\text { (ej.: lectura de libros, revistas, periódico; ver televisión; escuchar radio } \\
\text { u otros medios de audio; navegar o consultar información en Internet). }\end{array}$ & 58.9 & 59.5 & 58.5 \\
\hline Datos: Encuesta Nacional sobre Uso del Tiempo 2009, InEGl. & & \\
\hline
\end{tabular}

Haciendo un análisis más detallado de los medios masivos, estimamos que el $68.2 \%$ de las horas se asignaron a ver la TV; el $11.5 \%$ fueron dedicadas a escuchar radio u otros medios de audio, sin hacer otra actividad; el $10.7 \%$ se ocuparon en leer libros, revistas, periódicos u otros materiales impresos; mientras que un $9.6 \%$ fue destinado a navegar o realizar consultas de información por Internet.

Tabla 4. Demanda de medios masivos por bien

\begin{tabular}{|l|l|l|l|l|l|l|l|l|l|}
\hline \multicolumn{2}{|l|}{ Total de horas } & \multicolumn{3}{l|}{ Horas de lectura } & \multicolumn{2}{l|}{ Horas de TV } & \multicolumn{2}{l|}{ Horas de audio } & \multicolumn{2}{l|}{ Horas de Internet } \\
\hline Cantidad & $\%$ & Cantidad & $\%$ & Cantidad & $\%$ & Cantidad & $\%$ & Cantidad & $\%$ \\
\hline 483800.3 & 100 & 51940.7 & 10.7 & 329802.6 & 68.2 & 56030.15 & 11.5 & 46026.8 & 9.6 \\
\hline
\end{tabular}

En cuanto al consumo de televisión, mientras que el 68.2\% (Tabla 4) de horas dedicado a los medios masivos se pasan viendo TV, únicamente el 
27.7\% (Tabla 2) de los consumidores tienen conexión a la televisión por cable. De acuerdo con el Censo 2010 (INEGI, 2011), en este año el 94.7\% de los hogares poseían una TV. Esta evidencia permite inferir que una alta proporción de consumidores de TV sólo ven canales transmitidos a través de las frecuencias de VHF y UHF. Éstas son propiedad del Estado, están administradas por el gobierno y se encuentran concesionadas a las empresas Televisa y TV Azteca, aunque recientemente ha sido aprobada la reforma en el sector de telecomunicaciones que establece la posibilidad de que el gobierno haga nuevas concesiones.

Por otra parte, utilizando la Encuesta Nacional de Ingreso y Gasto de los Hogares (ENIGH) de 2010, se estimó la demanda de productos que son tradicionalmente ofrecidos por la industria editorial y de la música conforme al lugar de compra. Se encontró que el soporte de papel es todavía importante en México, así como la evidencia de piratería analógica: el 65.2\% de libros y $42.9 \%$ de las revistas fueron comprados en tiendas especializadas como las librerías; mientras que el $64.9 \%$ de los periódicos y el $26.6 \%$ de las revistas fueron adquiridas en kioscos o por los voceadores de la calle. En cuanto a los productos de audio, el $34.2 \%$ fue comprado principalmente a vendedores ambulantes y el $22.4 \%$ en los tianguis. Empero, el audio comprado en la calle era música pirata grabada en CD. Por su parte, el 38.6\% de los videojuegos fueron comprados en tiendas especializadas. Es muy importante notar el bajo porcentaje en las transacciones hechas por Internet: el 0.8 de libros, el 0.3 de revistas, y menos del 0.1 de periódicos y música.

Tabla 5. Demanda de productos impresos y grabados por lugar de compra (\%)

\begin{tabular}{|l|l|l|l|l|l|}
\hline \multicolumn{5}{|l|}{ Productos } \\
\hline Lugar de compra & Libros & Periódicos & Revistas & Audio & Videojuegos \\
\hline Mercado & 1.2 & 1.4 & 3.02 & 9.5 & 5.7 \\
\hline $\begin{array}{l}\text { Tianguis o mercado sobre } \\
\text { ruedas }\end{array}$ & 3.6 & 0.3 & 1.6 & 22.4 & 10.2 \\
\hline Vendedores ambulantes & 8.8 & 64.9 & 26.6 & 34.2 & 7.9 \\
\hline Tienda de abarrotes & 0.2 & 7.8 & 6.7 & 0.5 & 0 \\
\hline Tiendas específicas del ramo & 65.2 & 19.2 & 42.9 & 19.1 & 38.6 \\
\hline Supermercado & 3.8 & 0.7 & 14.1 & 6.9 & 12.5 \\
\hline Tienda departamental & 6.9 & $>0.1$ & 2.1 & 2.4 & 13.6 \\
\hline Compras fuera del país & 0.4 & $>0.1$ & 0.2 & 0.2 & 3.4 \\
\hline Tienda con membresía & 0.8 & $>0.1$ & 0.2 & 0.5 & 5.7 \\
\hline Persona particular & 8.02 & 5.3 & 1.9 & 3.9 & 2.3 \\
\hline Internet & 0.8 & $>0.1$ & 0.3 & $>0.1$ & 0 \\
\hline Datos: Encuesta Nacional de Ingreso y Gasto de los Hogares 2010 (ENIGH), INEGI. & \\
\hline
\end{tabular}


En 2005, la Organización para la Cooperación y el Desarrollo Económicos (OCDE) estimó que el comercio internacional de productos piratas alcanzó un valor de 2000 millones de dólares, excluyendo productos digitales. El $1^{\circ}$ de octubre de 2011 los estados miembros de la Unión Europea, Estados Unidos, Australia, Canadá, Corea del Sur, Japón, México, Marruecos, Nueva Zelanda, Singapur y Suiza firmaron el Acuerdo Comercial Antifalsificación (ACTA por sus siglas en inglés), con la intención de combatir a nivel internacional la violación de los derechos de propiedad intelectual (DPI), en particular la falsificación y la piratería, pretendiendo así acelerar la persecución de la violación de los DPI. Una vez que el acuerdo entre en vigor, cualquier país miembro de la Organización Mundial del Comercio (OMC) podrá unirse.

El 4 de julio de 2012, 478 Estados miembros del Parlamento Europeo votaron en contra de ACTA, 39 a favor y 165 se abstuvieron, lo que significó que el Acuerdo no entraría en vigor en la Unión Europea. Los opositores estaban preocupados de que ACTA favoreciera a las grandes corporaciones de los medios, en detrimento de los derechos ciudadanos. En mayo, la Comisión Europea refirió a ACTA a la Corte Europea de Justicia para su calificación y pidió al Parlamento esperar las conclusiones. De forma simultánea, el Parlamento decidió continuar adelante con su propio escrutinio del Acuerdo. Cinco comités se opusieron a ACTA, mientras que el comité revisor recibió una petición firmada por cerca de 3 millones de personas. ${ }^{12}$

En México, la legislación que gobierna la propiedad intelectual se encuentra en la Ley Federal de la Propiedad Industrial (LFPI), que entró en vigencia en 1991. Su precedente inmediato es la Ley para la Promoción y Protección de la Propiedad Industrial, una legislación que se consideraba en concordancia con los estándares internacionales de la época. La LFPI regula las patentes y los diseños industriales, los secretos comerciales, las marcas, los eslóganes publicitarios, nombres comerciales, denominaciones de origen y diseños de circuitos integrados. Asimismo, establece los procedimientos administrativos que pretenden proteger y preservar los derechos de la propiedad industrial y que permiten a la autoridad imponer medidas provisionales destinadas a la suspensión inmediata ante comportamientos violatorios de la ley.

Las áreas dedicadas a la protección de los derechos de autor se encuentran actualmente gobernadas por la Ley Federal de Derechos de Autor (LFDA), que entró en vigor en 1996 en sustitución de la ley del mismo nombre de 1956. Esta legislación contiene provisiones relacionadas con los derechos de autor en sí mismos, los derechos económicos y morales de los autores, la transferencia de

12 European Parliament, "Everything You Need to Know about ACTA", Focus (2012). Reference No. 0120220FCS38611, http://www.europarl.europa.eu. 
derechos económicos, la protección de derechos de autor y demás derechos relacionados. También establece directrices en relación con los derechos de autor de los símbolos patrios y las expresiones de la cultura popular, registro de derechos, reserva de derechos exclusivos, y la administración colectiva de derechos; crea el Instituto Nacional de Derechos de Autor (Indautor) y establece los procedimientos judiciales, conciliatorios y de arbitraje relacionados con el uso comercial de dichos derechos.

El 15 de julio de 2006, la Procuraduría General de la República (PGR) en conjunción con Televisa, la Business Software Association (BSA) y la Motion Picture Export Association of America (MPEAA) firmaron el Acuerdo Nacional contra la Piratería. La legislación mexicana establece conductas criminales que son merecedoras de multas o cárcel en relación con la propiedad intelectual. Sin embargo, con el objetivo de cumplir los acuerdos firmados ante la OMC, el Estado mexicano decidió reformar la legislación para asegurarse que la violación a los derechos de autor sean considerados una falta grave, para lo cual aumentó las penas y eliminó la posibilidad de fianza. ${ }^{13}$

El 11 de julio de 2012, el embajador de México ante Japón firmó el ACTA. Las razones por las que México no había firmado el acuerdo fueron que el Congreso de la Unión lo había rechazado, por lo que el Poder Ejecutivo buscó su aprobación recurriendo al Senado. Desde que México se unió a la OMC en enero de 1995, el gobierno federal ha apoyado continuamente los acuerdos de protección a la propiedad intelectual. Las compañías transnacionales han tenido un fiel aliado (aunque en ocasiones poco efectivo) en las agencias de procuración de justicia mexicanas. Igualmente, en apoyo a la protección de la propiedad intelectual, México firmó el Acuerdo de Aspectos relacionados con la Propiedad Intelectual (TRIP, por sus siglas en inglés) y el Congreso aprobó la legislación derivada de dichos acuerdos antes de 1995.

Tabla 6. Delitos en contra de la propiedad Intelectual

\begin{tabular}{|c|c|c|c|c|c|c|c|}
\hline \multirow[b]{2}{*}{ Concepto } & \multicolumn{4}{|c|}{ Datos anuales } & \multicolumn{2}{|c|}{ Enero-junio } & \multirow[b]{2}{*}{ Camb. (\%) } \\
\hline & 2007 & 2008 & 2009 & 2010 & 2010 & 2011 & \\
\hline $\begin{array}{l}\text { Operativos } \\
\text { y cateos }\end{array}$ & 1692 & 1990 & 1897 & 1666 & 782 & 677 & -13.4 \\
\hline Detenidos & 296 & 456 & 827 & 929 & 391 & 1,065 & 172.4 \\
\hline $\begin{array}{l}\text { Productos } \\
\text { apócrifos } \\
\text { asegurados }\end{array}$ & 140063138 & $\begin{array}{r}68820 \\
254\end{array}$ & 39221420 & 105328083 & 12714931 & 9168085 & -27.9 \\
\hline $\begin{array}{l}\text { Laboratorios } \\
\text { desmantelados }\end{array}$ & 206 & 217 & 200 & 91 & 61 & 78 & 27.9 \\
\hline
\end{tabular}

13 Procuraduría General de la República, Quinto informe de labores, México, 2011. 
En un ambiente de brecha digital, donde la mayoría de la piratería es realizada utilizando copias duras (DVD, CD y memorias USB), las acciones tomadas por la PGR han consistido en redadas policiacas, siguiendo la vieja escuela, que no requieren de aplicaciones de alta tecnología. Entre 2007 y 2010 la PGR realizó 7242 redadas; arrestó a 2508 personas; confiscó 353432865 copias de productos piratas y desmanteló 714 laboratorios (Tabla 7). A pesar de estos números, en apariencia impresionantes, no hay manera de realizar una evaluación apropiada de la efectividad e impacto de tales medidas. El volumen total de las actividades de la piratería permanece desconocido. Al mismo tiempo, dado el bajo costo de realizar copias digitales, es altamente probable que tan pronto es cerrado un laboratorio, otro más abra para llenar el sitio dejado por el anterior. En consecuencia, las medidas de alta tecnología, como el Manejo de Derechos Digitales (DRM, por sus siglas en inglés), parecen altamente improbables de ser efectivas. Las compañías propietarias pierden el control del contenido tan pronto como sus productos son "craqueados" y puestos a la venta a través de copias fuera de línea (offline) sin pasar por Internet.

Tabla 7. Productos apócrifos confiscados

\begin{tabular}{|l|r|r|r|r|r|r|r|r|}
\hline \multirow{2}{*}{ Concepto } & \multicolumn{2}{|c|}{2007} & \multicolumn{2}{c|}{2008} & \multicolumn{2}{c|}{2009} & \multicolumn{2}{c|}{2010} \\
\cline { 2 - 9 } & Cantidad & \multicolumn{1}{c|}{$\%$} & Cantidad & \multicolumn{1}{c|}{$\%$} & Cantidad & $\%$ & Cantidad & \multicolumn{1}{c|}{$\%$} \\
\hline DVD & 692435 & 29.4 & 2283632 & 14.6 & 1783122 & 37.4 & 2025024 & 34.5 \\
\hline Videojuegos & 28554 & 0.8 & 270120 & 1.7 & 77399 & 1.6 & 239192 & 4.1 \\
\hline Fonogramas (CD) & 2328807 & 62.2 & 1850406 & 11.8 & 1329790 & 27.9 & 1399820 & 23.8 \\
\hline Accesorios de vestir & 115946 & 3.2 & 143281 & 0.9 & 1328135 & 27.8 & 1433271 & 24.4 \\
\hline Calzado (pares) & 48519 & 1.4 & 6941 & 0.04 & 236536 & 4.9 & 737080 & 12.6 \\
\hline Medicamentos & 356447 & 10.0 & 1102550 & 19.49 & 7555 & 0.1 & 31466 & 0.5 \\
\hline TOTAL & 3570708 & 100.0 & 5656930 & 100 & 4762537 & 100 & 5865853 & 100 \\
\hline Fuente: Procuraduría General de la República, Quinto informe de labores, 2011. & \multicolumn{7}{|c|}{} \\
\hline
\end{tabular}

No obstante la gran dificultad para realizar una evaluación rigurosa de las actividades policiacas de la PGR en materia de derechos de autor, sus confiscaciones permiten hacer inferencias sobre la proporción del comercio pirata. La mayor proporción de productos piratas corresponde a copias duras de música y cine, CD y DVDS, seguido de medicinas, ropa y videojuegos, estos últimos tres en muy bajas proporciones (Tabla 7). La más alta proporción de CD y DVD, así como la proporción más baja de videojuegos confiscados, es consistente con las estimaciones de la Tabla 4.

En junio de 2000, Australia inquirió a la Misión Permanente de México ante el Consejo de TRIP en la OMC sobre si había alguna excepción en la Ley de Derechos de Autor en México que permitiera utilizar material protegido 
con fines de investigación, educación o uso legítimo o razonable (fair use). La delegación australiana preguntó si existe alguna decisión judicial, jurisprudencia relacionada o limitante de la ley que concierna a los derechos de autor relativos al software. ${ }^{14}$

La Misión Permanente de México ante el Consejo de TRIP respondió que bajo la Ley Federal de Derechos de Autor, Título VI, Art. 147, se establecen las limitantes a los derechos de autor y derechos conexos. En particular se refiere a las limitaciones por causa de utilidad pública. Hasta la fecha este artículo no ha sido aplicado:

Artículo 147.- Se considera de utilidad pública la publicación o traducción de obras literarias o artísticas necesarias para el adelanto de la ciencia, la cultura y la educación nacionales. Cuando no sea posible obtener el consentimiento del titular de los derechos patrimoniales correspondientes, y mediante el pago de una remuneración compensatoria, el Ejecutivo Federal, por conducto de la Secretaría de Educación Pública, de oficio o a petición de parte, podrá autorizar la publicación o traducción mencionada. Lo anterior será sin perjuicio de los tratados internacionales sobre derechos de autor y derechos conexos suscritos y aprobados por México.

\section{Conclusiones}

A lo largo de este artículo se argumenta que la presencia de la piratería digital es una actividad empresarial que depende de la evaluación racional de costos y beneficios derivada de respetar o no la ley de derechos de autor. Asimismo, que mientras exista la promesa de ganancias monopólicas otorgadas por los derechos de autor, la industria establecida tendrá la estrategia dominante de combatir la piratería o exigir al gobierno que lo haga, así como promover acuerdos internacionales que hagan valer sus derechos. No obstante, en comunidades en situación de pobreza digital las microempresas de piratería son uno de los canales mediante los cuales los consumidores tienen acceso a las industrias de la información.

En este sentido, los derechos de autor y el derecho a la información deberían considerarse complementarios, con posibilidades de actuar coordinada y sincrónicamente.

Esta doble dimensión teórico-práctica entre los dos tipos de derechos es una solución a los problemas del derecho de autor que suscita el ciberes-

14 World Trade Organization, Review of Legislation Mexico, Permanent Mission of Mexico, Council for Trade-Related Aspects of Intellectual Property Rights, Council Meeting 26 to 29 of June 2000. 
pacio pues posibilita la conjugación de ambos dentro del principio general de justicia que representa el derecho a la información. Extender el derecho a la información a los derechos de autor ofrece soluciones a los problemas derivados de Internet, bajo la convicción fundada de que las soluciones son justas en cada momento y situación frente a las dudas teórico-prácticas que surgen en la interpretación de la ley frente a lo que se considera abusos de los derechos de autor. ${ }^{15}$ En México, la brecha digital obstaculiza la oportunidad brindada por las tecnologías de la información para cumplir con el derecho a la información, a la vez que incentiva la piratería digital offline. Una política de conectividad sería mucho más efectiva que la persecución policiaca contra los piratas.

Las tecnologías digitales ofrecen posibilidades sin precedentes a la creatividad humana, la comunicación global y el acceso a la información. Sin embargo, en la década pasada las compañías de medios masivos desarrollaron métodos de control que afectan el derecho fundamental de uso, intercambio y reproducción de información e ideas. Estas tecnologías, al combinarse con las estructuras oligopólicas de la industria de medios y leyes, que buscan limitar el acceso valiéndose de la protección a los derechos de autor, amenazan la creatividad, privacidad y libertad de expresión de los ciudadanos. En cualquier caso, el gobierno debe intervenir no sólo para hacer cumplir la ley, sino para asegurar la salvaguarda de los derechos ciudadanos.

En los países desarrollados, las bibliotecas, organizaciones civiles y académicos han aplicado la idea de comunes de información a través de una diversidad de recursos informáticos abiertos y democráticos. Esto incluye comunes de software, licencias comunes, revistas académicas abiertas, repositorios digitales, etc. Las características básicas de estos comunes es que son colaborativos e interactivos, aprovechan el medio interconectado para construir comunidades de información beneficiándose de las externalidades de red, lo que significa que mientras más grande sea la participación, más valioso es el recurso. Muchas son libres y gratis, y su gobernabilidad es compartida con reglas y normas que son definidas y aceptadas por sus miembros, favoreciéndose así la libertad de expresión. ${ }^{16}$

En la práctica, las comunidades de información unen a personas alrededor de un interés común, lo que incrementa el acceso a un conjunto de recursos informativos. Internet suele ser uno de los principales nodos de estas comunidades, facilitando la conexión y la colaboración entre los participan-

15 J. M. Guanter Desantes, "Los derechos de autor en los medios ciberespaciales", Investigación Bibliotecológica 12 , no. 25 (1998): 17-32

16 N. Kranich, "The Information Commons: A Public Policy Report" (Brennan Center for Justice, NYU School of Law, 2004). 
tes, el intercambio de ideas y la vinculación con otros que tienen intereses y necesidades similares. Los académicos describen cinco características de estas comunidades de información basadas en el Internet:

1. Compartición de información con efectos múltiples.

2. Colaboración.

3. Interacción basada en las necesidades de los participantes.

4. Bajas barreras de entrada.

5. Conectividad con una comunidad de gran tamaño.

\section{Referencias}

Alexander, P. "Entry Barriers, Release Behavior, and Multi-Product Firms in the Music Recording Industry". Review of Industrial Organization 9 (1994): 85-98.

—."Peer-to-Peer File Sharing: The Case of the Music Recording Industry". Review of Industrial Organization 20 (2002): 151-61.

Armendáriz Sánchez, S. "La información como industria". Investigación Bibliotecológica 12 (1992): 11-24.

Benkler, Y. The Wealth of Networks. How Social Production Transforms Markets and Freedom. New Haven, Connecticut: Yale University Press, 2006.

Bhattacharjee, S., R. Gopal, K. Lertwachara y J. Marsden. "Impact of Legal Threats on Online Music Sharing Activity: An Analysis of Music Industry Legal Actions". The Journal of Law and Economics 49, no. 1 (2006): 91-114.

Caldera, J. y P. Arranz, "Cambios en los métodos de selección documental en los sistemas de información digital en las televisiones". Investigación Bibliotecológica 27, no. 60 (2013): 15-26.

Cossío Díaz, J. R. Derecho y análisis económico. México: Fondo de Cultura Económica, 2014.

Chávez, M., A. Santos, A. Figueroa, M. Ojeda e I. López. Panorama actual y retos para el archivamiento, intercambio y comercio de música digital. Mimeo, Universidad de la Sierra Sur, 2012.

Desantes, Guanter J. M. "Los derechos de autor en los medios ciberespaciales”. Investigación Bibliotecológica 12, no. 25 (1998): 17-32.

Dugatkin, L. A. y H. K. Reeve. Game Theory and Animal Behavior. Oxford: Oxford University Press, 1998.

European Parliament. "Everything You Need to Know about ACTA", Focus, 2012. Reference No. 20120220FCS38611. http://www. europarl.europa.eu.

Guallar, J., E. Abadal y Ll. Codina. "Sistemas de acceso a la información de prensa digital: tipología y evolución”. Investigación Bibliotecológica 27, no. 61 (2013): 29-52. 
Hess, C. y E. Ostrom. Understanding Knowledge as a Common. From Theory to Practice. Cambridge, Massachusetts: The MIT Press, 2007.

Hughes, J., M. Moore y E. Snyder. “'Napsterizing’ Pharmaceuticals: Access, Innovation, and Consumer Welfare”. National Bureau of Economic Research Working Paper Series, no. 9229 (2002).

Kranich, N. "The Information Commons: A Public Policy Report", the Free Expression. Policy Project, Brennan Center for Justice, NYU School of Law, 2004.

Kreps, D. y R. Wilson. "Reputation and Imperfect Information”. Journal of Economic Theory 27 (1982): 253-279.

Lessig, L. The Future of Ideas. The Fate of the Commons in a Connected World. Nueva York: Random House, 2001.

Liebowitz, S. J. "File Sharing: Creative Destruction or Just Plain Destruction?". The Journal of Law and Economics 49, no.1 (2006): 1-28.

Ostrom, E. Understanding Institutional Diversity. Princeton: Princeton University Press, 2005.

—. "Beyond Markets and States: Polycentric Governance of Complex Economic Systems”. American Economic Review 100 (2010): 64172.

Ostrom, E., R. Gardner y J. Walker. Rules, Games E Common-Pool Resources. Ann Arbor, Michigan: The University of Michigan Press, 2006.

Procuraduría General de la República (PGR). Quinto informe de labores. México: PGR, 2011.

Riley, J. Strong. "Evolutionary Equilibrium and the War of Attrition”. Journal of Theoretical Biology 82 (1980): 383-400.

Rob, R. y J. Waldfogel. "Piracy on the High C's: Music Downloading, Sales Displacement, and Social Welfare in A Sample of College Students". Journal of Law and Economics 49, no. 1 (2006): 29-62.

Sakakeeny, M. "Instruments of Power: Brass Bands in the Streets of New Orleans”. Tesis doctoral, Columbia University, 2008.

Secretaría de Seguridad Pública. Glosa del primer informe de Gobierno. Comparecencia Secretario de Seguridad Pública. H. Congreso del Estado, LXI Legislatura. Oaxaca. Última modificación en diciembre 9 de 2011. http://www.congresooaxaca.gob.mx/lxi/verstenograf.html.

Smith, J. M. Evolution and the Theory of Games. Cambridge: Cambridge University Press, 1982.

"The Theory of Games and the Evolution of Animal Conflict". Journal of Theoretical Biology 47 (1974): 209-21.

Tirole, J. The Theory of Industrial Organization. Cambridge, Massachusetts: The MIT Press, 1995.

U.S. Senate Committee on Commerce, Science and Transportation. The Future of Journalism. Hearing May 6, 2009. (SR-253). Washington D.C. http://commerce.senate.gov. 
World Trade Organization. Review of Legislation Mexico. Permanent Mission of Mexico. Council for Trade-Related Aspects of Intellectual Property Rights. Council meeting 26 to 29 of June 2000.

Zentner, A. "Measuring the Effect of File Sharing on Music Purchases". Journal of Law and Economics 49, no. 1 (2006): 63-90.

Para citar este artículo:

Chávez Ángeles, Manuel Gerardo y Patricia S. Sánchez Medina. "Industria de la información y piratería digital en México: análisis económico de la protección de los derechos de autor". Investigación Bibliotecológica 31, no. 71 (enero-abril 2017): 53-72.

DOI: http://dx.doi.org/10.22201/iibi.0187358xp.2017.71.57809

$\infty$ 